\title{
Existence Results for Nonlinear Anisotropic Elliptic Equation
}

\author{
Youssef Akdim ${ }^{\Uparrow}{ }^{1}$, Mostafa El moumni ${ }^{2}$, Abdelhafid Salmani ${ }^{1}$ \\ ${ }^{1}$ Sidi Mohamed Ben Abdellah University, Mathematics Physics and Computer Science, LSI, FP, Taza, Morocco \\ ${ }^{2}$ Chouaib Doukkali University, Department of Mathematics, Faculty of Sciences El jadida, Morocco
}

A R T I C L E I N F O

Article history:

Received: 20 May, 2017

Accepted: 15 July, 2017

Online: 29 December, 2017

Keywords:

Anisotropic elliptic equations

Weak solutions

Nonlinear operators

\section{Introduction}

In this paper we study the existence of weak solutions to anisotropic elliptic equations with homogeneous Dirichlet boundary conditions of the type

$$
\left\{\begin{array}{cc}
A u+\sum_{i=1}^{N} g_{i}(x, u, \nabla u)+\sum_{i=1}^{N} H_{i}(x, \nabla u) & \\
=f-\sum_{i=1}^{N} \frac{\partial}{\partial x_{i}} k_{i} & \text { in } \Omega,
\end{array}\right.
$$

where $\Omega$ is a bounded open subset of $\mathbb{R}^{N}(N \geq 2)$ with Lipschitz continuous boundary. The operator $A u=$ $-\sum_{i=1}^{N} \frac{\partial}{\partial x_{i}} a_{i}(x, u, \nabla u)$ is a Leray-Lions operator such that the functions $a_{i}, g_{i}$ and $H_{i}$ are the Carathodory functions satisfying the following conditions for all $s \in \mathbb{R}, \xi \in \mathbb{R}^{N}, \xi^{\prime} \in \mathbb{R}^{N}$ and a.e. in $\Omega$ :

$$
\begin{gathered}
\sum_{i=1}^{N} a_{i}(x, s, \xi) \xi_{i} \geq \lambda \sum_{i=1}^{N}\left|\xi_{i}\right|^{p_{i},} \\
\left|a_{i}(x, s, \xi)\right| \leq \gamma\left[|s|^{\frac{p_{\infty}^{\prime}}{p_{i}^{\prime}}}+\left|\xi_{i}\right|^{p_{i}-1}\right], \\
\left(a_{i}(x, s, \xi)-a_{i}\left(x, s, \xi^{\prime}\right)\right)\left(\xi_{i}-\xi_{i}^{\prime}\right)>0 \quad \text { for } \xi_{i} \neq \xi_{i}^{\prime}, \\
g_{i}(x, s, \xi) s \geq 0, \\
\left|g_{i}(x, s, \xi)\right| \leq L(|s|)\left|\xi_{i}\right|^{p_{i}} \forall i=1, \ldots ., N,
\end{gathered}
$$

$$
\left|H_{i}(x, \xi)\right| \leq b_{i}\left|\xi_{i}\right|^{p_{i}-1},
$$

where $\lambda, \gamma, b_{i}$ are some positive constants, for $i=$ $1, \ldots, N$ and $L: \mathbb{R}^{+} \rightarrow \mathbb{R}^{+}$is a continuous and non decreasing function. The right hand side $f$ and $k_{i}$ for $i=$ $1, \ldots, N$ are functions belonging to $L^{p_{\infty}^{\prime}}(\Omega)$ and $L^{p_{i}^{\prime}(\Omega)}$ where $p_{i}^{\prime}=\frac{p_{i}}{p_{i}-1}, p_{\infty}^{\prime}=\frac{p_{\infty}}{p_{\infty}-1}$ with $p_{\infty}=\max \left\{\bar{p}^{*}, p_{+}\right\}$ where $p_{+}=\max \left\{p_{1}, \ldots, p_{N}\right\}, \bar{p}=\frac{1}{\frac{1}{N} \sum_{i=1}^{N} \frac{1}{p_{i}}}$ and $\bar{p} *=\frac{N \bar{p}}{N-\bar{p}}$.

Since the growth and the coercivity conditions of each $a_{i}$ for all $i=1, \ldots, N$ depend on $p_{i}$, we have need to use the anisotropic Sobolev space. We mention some papers on anisotropic Sobolev spaces (see e.g.[1]-[5]).

If $p_{i}=p$ for all $i=1, \ldots, N$, we refer some works such as by Guibé in [6], by Monetti and Randazzo in 77 and by Y. Akdim, A. Benkirane and M. El Moumni in [8].

In [3], L.Boccardo, T. Gallouet and P. Marcellini have studied the problem (1) when $a_{i}(x, u, \nabla u)=$ $\left|\frac{\partial u}{\partial x_{i}}\right|^{p_{i}-1} \frac{\partial u}{\partial x_{i}}, g_{i}=0, H_{i}=0, k_{i}=0$ and $f=\mu$ is Radon's measure. In [5], F. Li has proved the existence and regularity of weak solutions of the problem 11 with $g_{i}=0, H_{i}=0, k_{i}=0$ for all $i=1, \ldots, N$ and $f$ belongs to $L^{m}(\Omega)$ with $m>1$. In [9], R. Di Nardo and F. Feo have proved the existence of weak solution of the problem (1) when $g_{i}=0$ for all $i=1, \ldots, N$. In [10], we have proved the existence and uniqueness of weak solution of the problem [1] but when $A u=-\sum_{i=1}^{N} \frac{\partial}{\partial x_{i}} a_{i}(x, \nabla u)$ $\left(a_{i}\right.$ depending only on $x$ and $\nabla u$ ).

In this work, we prove the existence of weak solu-

${ }^{*}$ Corresponding Author: Youssef Akdim, FP , Taza, Morocco \& youssef.akdim@usmba.ac.ma 
tions of the problem (1), based on techniques related to that of Di castro in [11] and to the recent work's Di Nardo and F. Feo in [9].

\section{Preliminaries}

Let $\Omega$ be a bounded open subset of $\mathbb{R}^{N}(N \geq 2)$ with Lipschitz continuous boundary and let $1<p_{1}, \ldots, p_{N}<$ $\infty$ be $\mathrm{N}$ real numbers, $p^{+}=\max \left\{p_{1}, \ldots, p_{N}\right\}, p^{-}=$ $\min \left\{p_{1}, \ldots, p_{N}\right\}$ and $\vec{p}=\left(p_{1}, \ldots, p_{N}\right)$. The anisotropic Sobolev space (see [12])

$$
W^{1, \vec{p}}(\Omega)=\left\{u \in W^{1,1}(\Omega): \frac{\partial u}{\partial x_{i}} \in L^{p_{i}}(\Omega), i=1,2, \ldots, N\right\}
$$

is a Banach space with respect to norm

$$
\|u\|_{W^{1, \vec{p}}(\Omega)}=\|u\|_{L^{1}(\Omega)}+\sum_{i=1}^{N}\left\|\frac{\partial u}{\partial x_{i}}\right\|_{L^{p_{i}(\Omega)}} .
$$

The space $W_{0}^{1, \vec{p}}(\Omega)$ is the closure of $C_{0}^{\infty}(\Omega)$ with respect to this norm. We recall a Poincarr-type inequality. Let $u \in W_{0}^{1, \vec{p}}(\Omega)$ then there exists a constant $C_{p}$ such that (see[13])

$$
\|u\|_{L^{p_{i}(\Omega)}} \leq C_{p}\left\|\frac{\partial u}{\partial x_{i}}\right\|_{L^{p_{i}(\Omega)}} \text { for } i=1, \ldots, N .
$$

Moreover a Sobolev-type inequality holds. Let us denote by $\bar{p}$ the harmonic mean of these numbers, i.e. $\frac{1}{\bar{p}}=\frac{1}{N} \sum_{i=1}^{N} \frac{1}{p_{i}}$. Let $u \in W_{0}^{1, \vec{p}}(\Omega)$, then there exists (see [12]) a constant $C_{s}$ such that

$$
\|u\|_{L^{q}(\Omega)} \leq C_{s} \prod_{i=1}^{N}\left\|\frac{\partial u}{\partial x_{i}}\right\|_{L^{p_{i}}(\Omega)}^{\frac{1}{N}}
$$

Where $q=\bar{p}^{*}=\frac{N \bar{p}}{N-\bar{p}}$ if $\bar{p}<N$ or $q \in[1,+\infty[$ if $\bar{p} \geq N$. We recall the arithmetic mean: Let $a_{1}, \ldots, a_{N}$ be positive numbers, it holds

$$
\prod_{i=1}^{N} a_{i}^{\frac{1}{N}} \leq \frac{1}{N} \sum_{i=1}^{N} a_{i}
$$

Which implies by 2]

$$
\|u\|_{L^{q}(\Omega)} \leq \frac{C_{s}}{N} \sum_{i=1}^{N}\left\|\frac{\partial u}{\partial x_{i}}\right\|_{L^{p_{i}(\Omega)}} .
$$

When $\bar{p}<N$ hold, inequality (4) implies the continuous embedding of the space $W_{0}^{1, \vec{p}}(\Omega)$ into $L^{q}(\Omega)$ for every $q \in\left[1, \bar{p}^{*}\right]$. On the other hand the continuity of the embedding $W_{0}^{1, \vec{p}}(\Omega) \hookrightarrow L^{p^{+}}(\Omega)$ relies on inequality (1). Let us put $p_{\infty}:=\max \left\{\bar{p}^{*}, p^{+}\right\}$

Proposition 1 For $q \in\left[1, p_{\infty}\right]$ there is a continuous embedding $W_{0}^{1, \vec{p}}(\Omega) \hookrightarrow L^{q}(\Omega)$. If $q<p_{\infty}$ the embedding is compact.

\section{Assumptions and Definition}

We consider the following class of nonlinear anisotropic elliptic homogenous Dirichlet problems

$$
\left\{\begin{array}{c}
-\sum_{i=1}^{N} \frac{\partial}{\partial x_{i}} a_{i}(x, u, \nabla u)+\sum_{i=1}^{N} g_{i}(x, u, \nabla u)+ \\
\sum_{i=1}^{N} H_{i}(x, \nabla u)=f-\sum_{i=1}^{N} \frac{\partial}{\partial x_{i}} k_{i} \text { in } \Omega, \\
u=0 \quad \text { on } \partial \Omega,
\end{array}\right.
$$

where $\Omega$ is a bounded open subset of $\mathbb{R}^{N}(N \geq 2)$ with Lipschitz continuous boundary $\partial \Omega, 1<p_{1}, \ldots, p_{N}<\infty$. We assume that $a_{i}: \Omega \times \mathbb{R} \times \mathbb{R}^{N} \rightarrow \mathbb{R}, g_{i}: \Omega \times \mathbb{R} \times \mathbb{R}^{N} \rightarrow \mathbb{R}$ and $H_{i}: \Omega \times \mathbb{R}^{N} \rightarrow \mathbb{R}$ are Carathodory functions such that for all $s \in \mathbb{R}, \xi \in \mathbb{R}^{N}, \xi^{\prime} \in \mathbb{R}^{N}$ and a. e. in $\Omega$ :

$$
\begin{gathered}
\sum_{i=1}^{N} a_{i}(x, s, \xi) \xi_{i} \geq \lambda \sum_{i=1}^{N}\left|\xi_{i}\right|^{p_{i}}, \\
\left|a_{i}(x, s, \xi)\right| \leq \gamma\left[|s|^{\frac{p_{\infty}}{p_{i}^{\prime}}}+\left|\xi_{i}\right|^{p_{i}-1}\right],
\end{gathered}
$$

$$
\left(a_{i}(x, s, \xi)-a_{i}\left(x, s, \xi^{\prime}\right)\right)\left(\xi_{i}-\xi_{i}^{\prime}\right)>0 \quad \text { for } \xi_{i} \neq \xi_{i}^{\prime},
$$

$$
g_{i}(x, s, \xi) s \geq 0
$$

$$
\left|g_{i}(x, s, \xi)\right| \leq L(|s|)\left|\xi_{i}\right|^{p_{i}} \forall i=1, \ldots ., N,
$$

$$
\left|H_{i}(x, \xi)\right| \leq b_{i}\left|\xi_{i}\right|^{p_{i}-1}
$$

where $\lambda, \gamma, b_{i}$ are some positive constants, for $i=$ $1, \ldots, N$ and $L: \mathbb{R}^{+} \rightarrow \mathbb{R}^{+}$is a continuous and non decreasing function. Moreover, we suppose that

$$
f \in L^{p_{\infty}^{\prime}}(\Omega)
$$

$$
k_{i} \in L^{p_{i}^{\prime}}(\Omega) \text { for } i=1, \ldots, N \text {. }
$$

Definition 1 A function $u \in W_{0}^{1, \vec{p}}(\Omega)$ is a weak solution of the problem $\square$ if $\sum_{i=1}^{N} g_{i}(x, u, \nabla u) \in L^{1}(\Omega)$ and $u$ satisfies

$$
\sum_{i=1}^{N} \int_{\Omega}\left[a_{i}(x, u, \nabla u) \frac{\partial \varphi}{\partial x_{i}}+g_{i}(x, u, \nabla u) \varphi+H_{i}(x, \nabla u) \varphi\right]
$$

$$
=\int_{\Omega}\left[f \varphi+\sum_{i=1}^{N} k_{i} \frac{\partial \varphi}{\partial x_{i}}\right]
$$

$\forall \varphi \in W_{0}^{1, \vec{p}}(\Omega) \cap L^{\infty}(\Omega)$. 


\section{Main results}

In this section we prove the existence of at least a weak solution of the problem (1). We consider the approximate problems.

\subsection{Approximate problems and a priori estimates}

Let

$$
g_{i}^{n}(x, u, \nabla u)=\frac{g_{i}(x, u, \nabla u)}{1+\frac{1}{n}\left|g_{i}(x, u, \nabla u)\right|}
$$

and

$$
H_{i}^{n}(x, \nabla u)=\frac{H_{i}(x, \nabla u)}{1+\frac{1}{n}\left|H_{i}(x, \nabla u)\right|} .
$$

By Leray-Lions (see e.g. [14]), there exists at least a weak solution $u_{n} \in W_{0}^{1, \vec{p}}(\Omega)$ of the following approximate problem

$$
\left\{\begin{array}{c}
-\sum_{i=1}^{N} \frac{\partial}{\partial x_{i}} a_{i}\left(x, u_{n}, \nabla u_{n}\right)+\sum_{i=1}^{N} g_{i}^{n}\left(x, u_{n}, \nabla u_{n}\right) \\
+\sum_{i=1}^{N} H_{i}^{n}\left(x, \nabla u_{n}\right)=f-\sum_{i=1}^{N} \frac{\partial}{\partial x_{i}} k_{i} \text { in } \Omega \\
u_{n}=0 \quad \text { on } \partial \Omega .
\end{array}\right.
$$

Lemma 1 See ([9], lemma 4.2) Let $A \in \mathbb{R}^{+}$and $u \in$ $W_{0}^{1, \vec{p}}(\Omega)$, then there exists $t$ measurable subsets $\Omega_{1}, \ldots, \Omega_{t}$ of $\Omega$ and $t$ functions $u_{1}, \ldots, u_{t}$ such that $\Omega_{i} \cap \Omega_{j}=\emptyset$ for $i \neq j,\left|\Omega_{t}\right| \leq A$ and $\left|\Omega_{s}\right|=A$ for $s \in\{1, \ldots, t-1\},\{x \in \Omega$ : $\left|\frac{\partial u_{s}}{\partial x_{i}}\right| \neq 0 \quad$ for $\left.i=1, \ldots, N\right\} \subset \Omega_{s}, \frac{\partial u}{\partial x_{i}}=\frac{\partial u_{s}}{\partial x_{i}} \quad$ a. e. in $\Omega_{s}$, $\frac{\partial\left(u_{1}+\ldots+u_{s}\right)}{\partial x_{i}} u_{s}=\left(\frac{\partial u}{\partial x_{i}}\right) u_{s}, \quad u_{1}+\ldots+u_{s}=u$ in $\Omega$ and $\operatorname{sign}(u)=\operatorname{sign}\left(u_{s}\right)$ if $u_{s} \neq 0$ for $s \in\{1, \ldots, t\}$ and $i \in$ $\{1, \ldots, N\}$.

Proposition 2 Assume that $\bar{p}<N$, (5)-(12) hold and let $u_{n} \in W_{0}^{1, \vec{p}}(\Omega)$ be a solution to problem 13 then, we have

$$
\sum_{i=1}^{N} \int_{\Omega}\left|\frac{\partial u_{n}}{\partial x_{i}}\right|^{p_{i}} \leq C,
$$

for some positive constant $C$ depending on $N, \Omega, \lambda, \gamma, p_{i}$, $b_{i},\|f\|_{L^{p_{\infty}(\Omega)}},\left\|g_{i}\right\|_{L^{p_{i}(\Omega)}}$ for $i=1, \ldots, N$.

Proof: Let $A$ be a positive real number, that will be chosen later, Referring to lemma 1 . Let us fix $s \in\{1, \ldots, t\}$ and let us use $T_{k}\left(u_{s}\right)$ as test function in problem 13 . using (5), (8), Young's and Hölder's inequalities and proposition 1 we obtain

$$
\begin{gathered}
\sum_{i=1}^{N} \int_{\left\{u_{s} \leq k\right\}}\left|\frac{\partial u_{s}}{\partial x_{i}}\right|^{p_{i}} \\
\leq C_{1}\left(\|f\|_{L^{p_{\infty}^{\prime}(\Omega)}} d_{s}^{\frac{1}{N}}+\sum_{i=1}^{N} \int_{\Omega}\left|H_{i}(x, \nabla u)\left\|u_{s} \mid+\sum_{i=1}^{N}\right\| k_{i} \|_{L^{p_{i(\Omega)}^{\prime}}}^{p_{i}^{\prime}}\right) .\right.
\end{gathered}
$$


inequality 17 becomes

$\sum_{i=1}^{N} \int_{\Omega}\left|\frac{\partial u_{s}}{\partial x_{i}}\right|^{p_{i}} \leq C_{4}\left\{\|f\|_{L_{\infty}^{p_{\infty}(\Omega)}} d_{s}^{\frac{1}{N}}+\sum_{i=1}^{N}\left\|k_{i}\right\|_{L^{p_{i}^{\prime}(\Omega)}}^{p_{i}^{\prime}}+\right.$

$\left.\sum_{\sigma=1}^{s-1} \sum_{i=1}^{N} A^{\frac{1}{p_{i}}-\frac{1}{p_{\infty}}}\left(\sum_{j=1}^{N} \int_{\Omega_{s}}\left|\frac{\partial u_{s}}{\partial x_{j}}\right|^{p_{j}}\right)+\sum_{i=1}^{N} A^{\frac{1}{p_{i}}-\frac{1}{p_{\infty}}} d_{s}^{\frac{p_{i}}{N}}\right\}$

for some constant $C_{4}>0$ and for $s=1$, we get

$\int_{\Omega}\left|\frac{\partial u_{1}}{\partial x_{i}}\right|^{p_{i}} \leq \sum_{i=1}^{N} \int_{\Omega}\left|\frac{\partial u_{1}}{\partial x_{i}}\right|^{p_{i}}$

$\leq C_{4}\left\{\|f\|_{L^{p_{\infty}(\Omega)}} d_{1}^{\frac{1}{N}}+\sum_{i=1}^{N}\left\|k_{i}\right\|_{L^{p_{i}^{\prime}(\Omega)}}^{p_{i}^{\prime}}+\sum_{i=1}^{N} A^{\frac{1}{p_{i}}-\frac{1}{p_{\infty}}} d_{1}^{\frac{p_{i}}{N}}\right\}$.

Let us choose $A$ such that 18 and

$1-C_{4} \sum_{i=1}^{N} A^{\frac{N}{p_{i}}\left(\frac{1}{p_{i}}-\frac{1}{p_{\infty}}\right)}>0$ hold, (see [9]).

For example, we can take

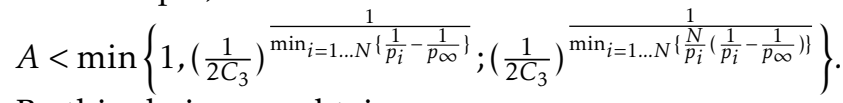
By this choice, we obtain

$d_{1}=\prod_{i=1}^{N}\left(\int_{\Omega}\left|\frac{\partial u_{1}}{\partial x_{i}}\right|^{p_{i}}\right)^{\frac{1}{p_{i}}}$

$\leq C_{5}\left[\left(\|f\|_{L^{p_{\infty}^{\prime}(\Omega)}}^{\frac{N}{\bar{p}}}+\left\|v_{2}\right\|_{L^{p_{\infty}(\Omega)}}^{\frac{N}{\bar{p}}}\right) d_{1}^{\frac{1}{\bar{p}}}+\sum_{i=1}^{N}\left\|k_{i}\right\|_{L^{p_{i(\Omega)}^{\prime}}}^{p_{i}^{\prime}}\right]$. Then

there exists a constant $C_{6}>0$ such that $d_{1} \leq C_{6}$ and by 20, we obtain

$$
\sum_{i=1}^{N} \int_{\Omega}\left|\frac{\partial u_{1}}{\partial x_{i}}\right|^{p_{i}} \leq C_{7}
$$

for some constant $C_{7}>0$. Moreover using 21) in (19) and iterating on $s$, we have

$\sum_{i=1}^{N} \int_{\Omega}\left|\frac{\partial u_{s}}{\partial x_{i}}\right|^{p_{i}}$

$\leq C_{8}\left[\|f\|_{L_{\infty}^{p_{\infty}(\Omega)}} d_{s}^{\frac{1}{N}}+\sum_{i=1}^{N}\left\|k_{i}\right\|_{L^{p_{i}^{\prime}(\Omega)}}^{p_{i}^{\prime}}+1+\sum_{i=1}^{N} A^{\frac{1}{p_{i}}-\frac{1}{p_{\infty}}} d_{s}^{\frac{p_{i}}{N}}\right]$

then arguing as before, we obtain $\sum_{i=1}^{N} \int_{\Omega}\left|\frac{\partial u_{s}}{\partial x_{i}}\right| p_{i} \leq C_{9}$, for some constant $C_{9}>0$, then

$\|u\|_{W_{0}^{1, \vec{p}}(\Omega)} \leq k \sum_{i=1}^{N}\left(\sum_{s=1}^{t} \int_{\Omega}\left|\frac{\partial u_{s}}{\partial x_{i}}\right|^{p_{i}}\right)^{\frac{1}{p_{i}}} \leq C_{10}$, for some positive $k>0$.

Since $u_{n}$ is bounded in $W_{0}^{1, \vec{p}}(\Omega)$ and the embed$\operatorname{ding} W_{0}^{1, \vec{p}}(\Omega) \hookrightarrow L^{p_{-}}(\Omega)$ is compact, we obtain the following results.

Corollaire 1 If $u_{n}$ is a weak solution of problem (13), then there exists a subsequence $\left(u_{n}\right)_{n}$ such that $u_{n} \rightarrow u$ weakly in $W_{0}^{1, \vec{p}}(\Omega)$, strongly in $L^{p_{-}}(\Omega)$ and a. e. in $\Omega$.

\subsection{Strong convergence of $T_{k}\left(u_{n}\right)$}

Lemma 2 Assume that $u_{n} \rightarrow u$ weakly in $W_{0}^{1, \vec{p}}(\Omega)$ and a. e. in $\Omega$ and

$\sum_{i=1}^{N} \int_{\Omega}\left[a_{i}\left(x, u_{n}, \nabla u_{n}\right)-a_{i}\left(x, u_{n}, \nabla u\right)\right]\left(\frac{\partial u_{n}}{\partial x_{i}}-\frac{\partial u}{\partial x_{i}}\right) \rightarrow 0$ then,

$$
u_{n} \rightarrow u \text { strongly in } W_{0}^{1, \vec{p}}(\Omega) \text {. }
$$

Proof: The proof follows as in Lemma 5 of [15] taking into account the anisotropy of operator.

Proposition 3 Let $u_{n}$ be a solution to the approximate problem (13), then

$$
T_{k}\left(u_{n}\right) \rightarrow T_{k}(u) \text { strongly in } W_{0}^{1, \vec{p}}(\Omega)
$$

Proof: Let us fix $k$ and let $\delta$ be a real number such that $\delta \geq\left(\frac{L(k)}{2 \lambda}\right)^{2}$. Let us define $z_{n}=T_{k}\left(u_{n}\right)-T_{k}(u)$ and $\varphi(s)=s e^{\delta s^{2}}$, it is easy to check that for all $s \in \mathbb{R}$ one has

$$
\varphi^{\prime}(s)-\frac{L(k)}{\lambda}|\varphi(s)| \geq \frac{1}{2} .
$$

Using $\varphi\left(z_{n}\right)$ as test function in 13, we get

$\sum_{i=1}^{N} \int_{\Omega} a_{i}\left(x, u_{n}, \nabla u_{n}\right) \frac{\partial \varphi\left(z_{n}\right)}{\partial x_{i}}$

$+\sum_{i=1}^{N} \int_{\Omega} g_{i}^{n}\left(x, u_{n}, \nabla u_{n}\right) \varphi\left(z_{n}\right)+\sum_{i=1}^{N} \int_{\Omega} H_{i}^{n}\left(x, \nabla u_{n}\right) \varphi\left(z_{n}\right)=$

$$
\int_{\Omega} f \varphi\left(z_{n}\right)+\sum_{i=1}^{N} \int_{\Omega} k_{i}(x) \frac{\partial \varphi\left(z_{n}\right)}{\partial x_{i}} .
$$

Since $\varphi\left(z_{n}\right) \rightarrow 0$ weakly in $W_{0}^{1, \vec{p}}(\Omega) \hookrightarrow L^{p_{\infty}}(\Omega)$ and $f \in L^{p_{\infty}^{\prime}}(\Omega)$ then $\int_{\Omega} f \varphi\left(z_{n}\right) \rightarrow 0$ as $n \rightarrow+\infty$. Since $T_{k}\left(u_{n}\right) \rightarrow T_{k}(u)$ weakly in $W_{0}^{1, \vec{p}}(\Omega), k_{i} \in L^{p_{i}^{\prime}}(\Omega)$ and $\left(\varphi^{\prime}\left(z_{n}\right)\right)_{n}$ is bounded then $\sum_{i=1}^{N} \int_{\Omega} k_{i}(x) \frac{\partial \varphi\left(z_{n}\right)}{\partial x_{i}} \rightarrow 0$ as $n \rightarrow+\infty$. On the other hand, we have

$$
\begin{aligned}
\mid \sum_{i=1}^{N} \int_{\Omega} H_{i}^{n}(x, & \left.\nabla u_{n}\right) \varphi\left(z_{n}\right) \mid \\
& \leq \sum_{i=1}^{N} \int_{\Omega}\left|\frac{\partial u_{n}}{\partial x_{i}}\right|^{p_{i}-1}\left|b_{i} \varphi\left(z_{n}\right)\right| \\
& \leq \sum_{i=1}^{N}\left(\int_{\Omega}\left|b_{i} \varphi\left(z_{n}\right)\right|^{p_{i}}\right)^{\frac{1}{p_{i}}}\left(\int_{\Omega}\left|\frac{\partial u_{n}}{\partial x_{i}}\right|^{p_{i}}\right)^{\frac{1}{p_{i}^{\prime}}} \\
& \leq C \sum_{i=1}^{N}\left(\int_{\Omega}\left|b_{i} \varphi\left(z_{n}\right)\right|^{p_{i}}\right)^{\frac{1}{p_{i}}} .
\end{aligned}
$$

Since $b_{i} \varphi\left(z_{n}\right) \rightarrow 0$ a.e. in $\Omega$ and $\left|b_{i} \varphi\left(z_{n}\right)\right| \leq$ $\left|b_{i}\right| \times 2 k e^{4 k^{2} \delta} \in L^{p_{i}}(\Omega)$, then by dominated convergence theorem $b_{i} \varphi\left(z_{n}\right) \rightarrow 0$ strongly in $L^{p_{i}}(\Omega)$, then $\left|\sum_{i=1}^{N} \int_{\Omega} H_{i}^{n}\left(x, \nabla u_{n}\right) \varphi\left(z_{n}\right) d x\right| \rightarrow 0$ as $n \rightarrow+\infty$. Denote by $\varepsilon_{1}(n), \varepsilon_{2}(n), \ldots .$. various sequences of real numbers which converge to zero when $n$ tends to $+\infty$. Using (8), we obtain that $g_{i}^{n}\left(x, u_{n}, \nabla u_{n}\right) \varphi\left(z_{n}\right) \geq 0$ on the set $\left\{\left|u_{n}\right|>k\right\}$. Then we have

$\sum_{i=1}^{N} \int_{\Omega} a_{i}\left(x, u_{n}, \nabla u_{n}\right) \frac{\partial \varphi\left(z_{n}\right)}{\partial x_{i}}+$

$\sum_{i=1}^{N} \int_{\left\{\left|u_{n}\right| \leq k\right\}} g_{i}^{n}\left(x, u_{n}, \nabla u_{n}\right) \varphi\left(z_{n}\right) d x \leq \varepsilon_{1}(n)$ 
On the other hand, we have

$\sum_{i=1}^{N} \int_{\Omega} a_{i}\left(x, u_{n}, \nabla u_{n}\right) \frac{\partial \varphi\left(z_{n}\right)}{\partial x_{i}}$

$=\sum_{i=1}^{N} \int_{\Omega} a_{i}\left(x, u_{n}, \nabla u_{n}\right)\left(\frac{\partial T_{k}\left(u_{n}\right)}{\partial x_{i}}-\frac{\partial T_{k}(u)}{\partial x_{i}}\right) \varphi^{\prime}\left(z_{n}\right)$

$=\sum_{i=1}^{N} \int_{\Omega} a_{i}\left(x, T_{k}\left(u_{n}\right), \nabla T_{k}\left(u_{n}\right)\right)\left(\frac{\partial T_{k}\left(u_{n}\right)}{\partial x_{i}}-\frac{\partial T_{k}(u)}{\partial x_{i}}\right) \varphi^{\prime}\left(z_{n}\right)$

$-\sum_{i=1}^{N} \int_{\left\{u_{n}>k\right\}} a_{i}\left(x, u_{n}, \nabla u_{n}\right) \frac{\partial T_{k}(u)}{\partial x_{i}} \varphi^{\prime}\left(z_{n}\right)$.

The sequence $\left(a_{i}\left(x, u_{n}, \nabla u_{n}\right) \varphi^{\prime}\left(z_{n}\right)\right)_{n}$ is bounded in

$L^{p_{i}^{\prime}}(\Omega)$, then since $\frac{\partial T_{k}(u)}{\partial x_{i}} \chi_{\left\{\left|u_{n}\right|>k\right\}} \rightarrow 0$ strongly in $L^{p_{i}}(\Omega)$,

one has

$\sum_{i=1}^{N} \int_{\Omega} a_{i}\left(x, u_{n}, \nabla u_{n}\right) \frac{\partial \varphi\left(z_{n}\right)}{\partial x_{i}} d x$

$=\sum_{i=1}^{N} \int_{\Omega} a_{i}\left(x, T_{k}\left(u_{n}\right), \nabla T_{k}\left(u_{n}\right)\right)\left(\frac{\partial T_{k}\left(u_{n}\right)}{\partial x_{i}}-\frac{\partial T_{k}(u)}{\partial x_{i}}\right) \varphi^{\prime}\left(z_{n}\right)$

$+\varepsilon_{2}(n)$

which we can write

$\sum_{i=1}^{N} \int_{\Omega} a_{i}\left(x, u_{n}, \nabla u_{n}\right) \frac{\partial \varphi\left(z_{n}\right)}{\partial x_{i}} d x$

$=\sum_{i=1}^{N} \int_{\Omega}\left(a_{i}\left(x, T_{k}\left(u_{n}\right), \nabla T_{k}\left(u_{n}\right)\right)-a_{i}\left(x, T_{k}\left(u_{n}\right), \nabla T_{k}(u)\right)\right)$

$\left(\frac{\partial T_{k}\left(u_{n}\right)}{\partial x_{i}}-\frac{\partial T_{k}(u)}{\partial x_{i}}\right) \varphi^{\prime}\left(z_{n}\right) d x$

$+\sum_{i=1}^{N} \int_{\Omega} a_{i}\left(x, T_{k}\left(u_{n}\right), \nabla T_{k}(u)\right)\left(\frac{\partial T_{k}\left(u_{n}\right)}{\partial x_{i}}-\frac{\partial T_{k}(u)}{\partial x_{i}}\right) \varphi^{\prime}\left(z_{n}\right) d x$

$+\varepsilon_{2}(n)$.

Since $u_{n} \rightarrow u$ a.e. in $\Omega$, we have

$a_{i}\left(x, T_{k}\left(u_{n}\right), \nabla T_{k}(u)\right) \varphi^{\prime}\left(z_{n}\right)$ converges to

$a_{i}\left(x, T_{k}(u), \nabla T_{k}(u)\right)$ a. e. in $\Omega$. Let $E$ be measurable subset of $\Omega$, by the growth condition (6), we get

$\int_{E}\left|a_{i}\left(x, T_{k}\left(u_{n}\right), \nabla T_{k}(u)\right) \varphi^{\prime}\left(z_{n}\right)\right|^{p_{i}^{\prime}} d x$

$\leq 2^{p_{i}^{\prime}-1}\left(1+8 \delta k^{2}\right)^{p_{i}^{\prime}} e^{4 \delta k^{2} p_{i}^{\prime}}\left(k^{p_{\infty}}|E|+\int_{E} \mid \frac{\partial T_{k}(u)}{\partial x_{i}} p^{p_{i}}\right)$.

Then the sequence $\left(a_{i}\left(x, T_{k}\left(u_{n}\right), \nabla T_{k}(u)\right) \varphi^{\prime}\left(z_{n}\right)\right)_{n}$ is equi-integrable and by Vitali's theorem one has $a_{i}\left(x, T_{k}\left(u_{n}\right), \nabla T_{k}(u)\right) \varphi^{\prime}\left(z_{n}\right)$ converges to $a_{i}\left(x, T_{k}(u), \nabla T_{k}(u)\right) \quad$ strongly in $L^{p_{i}^{\prime}}(\Omega)$. Since $\frac{\partial T_{k}\left(u_{n}\right)}{\partial x_{i}} \rightarrow \frac{\partial T_{k}(u)}{\partial x_{i}} \quad$ weakly in $L^{p_{i}}(\Omega)$, then $\lim _{n \rightarrow+\infty} \sum_{i=1}^{N} \int_{\Omega} a_{i}\left(x, T_{k}\left(u_{n}\right), \nabla T_{k}(u)\right)$

$\left(\frac{\partial T_{k}\left(u_{n}\right)}{\partial x_{i}}-\frac{\partial T_{k}(u)}{\partial x_{i}}\right) \varphi^{\prime}\left(z_{n}\right)=0$. It follows that

$\sum_{i=1}^{N} \int_{\Omega} a_{i}\left(x, u_{n}, \nabla u_{n}\right) \frac{\partial \varphi\left(z_{n}\right)}{\partial x_{i}}$

$=\sum_{i=1}^{N} \int_{\Omega}\left(a_{i}\left(x, T_{k}\left(u_{n}\right), \nabla T_{k}\left(u_{n}\right)\right)-a_{i}\left(x, T_{k}\left(u_{n}\right), \nabla T_{k}(u)\right)\right)$

$$
\left(\frac{\partial T_{k}\left(u_{n}\right)}{\partial x_{i}}-\frac{\partial T_{k}(u)}{\partial x_{i}}\right) \varphi^{\prime}\left(z_{n}\right)+\varepsilon_{3}(n) .
$$

On the other hand, by virtue of (5) and (9)

$\left|\sum_{i=1}^{N} \int_{\left\{\left|u_{n}\right| \leq k\right\}} g_{i}^{n}\left(x, u_{n}, \nabla u_{n}\right) \varphi\left(z_{n}\right) d x\right|$

$\leq L(k) \sum_{i=1}^{N} \int_{\left\{\left|u_{n}\right| \leq k\right\}}\left|\frac{\partial u_{n}}{\partial x_{i}}\right|^{p_{i}}\left|\varphi\left(z_{n}\right)\right| d x$

$\leq L(k) \sum_{i=1}^{N} \int_{\Omega}\left|\frac{\partial T_{k} u_{n}}{\partial x_{i}}\right|^{p_{i}}\left|\varphi\left(z_{n}\right)\right| d x$

$\leq \frac{L(k)}{\lambda} \sum_{i=1}^{N} \int_{\Omega} a_{i}\left(x, T_{k}\left(u_{n}\right), \nabla T_{k}\left(u_{n}\right)\right) \cdot \frac{\partial T_{k}\left(u_{n}\right)}{\partial x_{i}}\left|\varphi\left(z_{n}\right)\right| d x$

$\leq \frac{L(k)}{\lambda} \sum_{i=1}^{N} \int_{\Omega}\left(a_{i}\left(x, T_{k}\left(u_{n}\right), \nabla T_{k}\left(u_{n}\right)\right)-\right.$

$\left.a_{i}\left(x, T_{k}\left(u_{n}\right), \nabla T_{k}(u)\right)\right)\left(\frac{\partial T_{k}\left(u_{n}\right)}{\partial x_{i}}-\frac{\partial T_{k}(u)}{\partial x_{i}}\right)\left|\varphi\left(z_{n}\right)\right| d x+$

$\frac{L(k)}{\lambda} \sum_{i=1}^{N} \int_{\Omega} a_{i}\left(x, T_{k}\left(u_{n}\right), \nabla T_{k}\left(u_{n}\right)\right) \cdot \frac{\partial T_{k}(u)}{\partial x_{i}}\left|\varphi\left(z_{n}\right)\right| d x+$

$\frac{L(k)}{\lambda} \sum_{i=1}^{N} \int_{\Omega} a_{i}\left(x, T_{k}\left(u_{n}\right), \nabla T_{k}(u)\right)$

$$
\cdot\left(\frac{\partial T_{k}\left(u_{n}\right)}{\partial x_{i}}-\frac{\partial T_{k}(u)}{\partial x_{i}}\right)\left|\varphi\left(z_{n}\right)\right| d x .
$$

Similarly as above, it's easy to see that by (6), corollary 1 and Vitali's theorem one has

$a_{i}\left(x, T_{k}\left(u_{n}\right), \nabla T_{k}(u)\right) \rightarrow a_{i}\left(x, T_{k}(u), \nabla T_{k}(u)\right)$ strongly in $L^{p_{i}(\Omega) \text {. Writing }}$

$\left|\sum_{i=1}^{N} \int_{\Omega} a_{i}\left(x, T_{k}\left(u_{n}\right), \nabla T_{k}(u)\right) \cdot\left(\frac{\partial T_{k}\left(u_{n}\right)}{\partial x_{i}}-\frac{\partial T_{k}(u)}{\partial x_{i}}\right) \varphi\left(z_{n}\right) d x\right|$ $\leq \varphi(2 k) \sum_{i=1}^{N} \int_{\Omega}\left|a_{i}\left(x, T_{k}\left(u_{n}\right), \nabla T_{k}(u)\right)\right| \cdot\left|\frac{\partial T_{k}\left(u_{n}\right)}{\partial x_{i}}-\frac{\partial T_{k}(u)}{\partial x_{i}}\right|$ and taking into account that $\frac{\partial T_{k}\left(u_{n}\right)}{\partial x_{i}} \rightarrow \frac{\partial T_{k}(u)}{\partial x_{i}}$ weakly in $L^{p_{i}}(\Omega)$, we obtain

$\sum_{i=1}^{N} \int_{\Omega} a_{i}\left(x, T_{k}\left(u_{n}\right), \nabla T_{k}(u)\right) \cdot\left(\frac{\partial T_{k}\left(u_{n}\right)}{\partial x_{i}}-\frac{\partial T_{k}(u)}{\partial x_{i}}\right) \varphi\left(z_{n}\right) d x$ tends to zero as $n \rightarrow+\infty$. Thanks to 6 and (14), the sequence $\left(a_{i}\left(x, T_{k}\left(u_{n}\right), \nabla T_{k}\left(u_{n}\right)\right)\right)_{n}$ is bounded in $L^{p_{i}^{\prime}}(\Omega)$, so that there exists $l_{k}^{i} \in L^{p_{i}^{\prime}}(\Omega)$ such that $a_{i}\left(x, T_{k}\left(u_{n}\right), \nabla T_{k}\left(u_{n}\right)\right) \rightarrow l_{k}^{i}$ weakly in $L^{p_{i}^{\prime}}(\Omega)$. We have

$\sum_{i=1}^{N} \int_{\Omega} a_{i}\left(x, T_{k}\left(u_{n}\right), \nabla T_{k}\left(u_{n}\right)\right) \frac{\partial T_{k}(u)}{\partial x_{i}} \varphi\left(z_{n}\right) d x=$ $\sum_{i=1}^{N} \int_{\Omega}\left(a_{i}\left(x, T_{k}\left(u_{n}\right), \nabla T_{k}\left(u_{n}\right)\right)-l_{k}^{i}\right) \frac{\partial T_{k}(u)}{\partial x_{i}} \varphi\left(z_{n}\right) d x$

$+\sum_{i=1}^{N} \int_{\Omega} l_{k}^{i} \frac{\partial T_{k}(u)}{\partial x_{i}} \varphi\left(z_{n}\right) d x$.

Since $\varphi\left(z_{n}\right) \rightarrow 0$ weak $^{*}$ in $L^{\infty}(\Omega)$, we conclude that $\sum_{i=1}^{N} \int_{\Omega} a_{i}\left(x, T_{k}\left(u_{n}\right), \nabla T_{k}\left(u_{n}\right)\right) \frac{\partial T_{k}(u)}{\partial x_{i}} \varphi\left(z_{n}\right) d x \rightarrow 0$ as $n \rightarrow$ $+\infty$. Hence, we get 


$$
\begin{aligned}
& \left|\sum_{i=1}^{n} \int_{\left\{\left|u_{n}\right| \leq k\right\}} g_{i}^{n}\left(x, u_{n}, \nabla u_{n}\right) \varphi\left(z_{n}\right) d x\right| \\
& \leq \frac{L(k)}{\lambda} \sum_{i=1}^{N} \int_{\Omega}\left(a_{i}\left(x, T_{k}\left(u_{n}\right), \nabla T_{k}\left(u_{n}\right)\right)\right. \\
& \left.-a_{i}\left(x, T_{k}\left(u_{n}\right), \nabla T_{k}(u)\right)\right)\left(\frac{\partial T_{k}\left(u_{n}\right)}{\partial x_{i}}-\frac{\partial T_{k}(u)}{\partial x_{i}}\right)\left|\varphi\left(z_{n}\right)\right| d x \\
& +\varepsilon_{5}(n) . \\
& \sum_{i=1}^{N} \int_{\Omega}\left(a_{i}\left(x, T_{k}\left(u_{n}\right), \nabla T_{k}\left(u_{n}\right)\right)-a_{i}\left(x, T_{k}\left(u_{n}\right), \nabla T_{k}(u)\right)\right) \\
& \left(\frac{\partial T_{k}\left(u_{n}\right)}{\partial x_{i}}-\frac{\partial T_{k}(u)}{\partial x_{i}}\right)\left(\varphi^{\prime}\left(z_{n}\right)-\frac{L(k)}{\lambda}\left|\varphi\left(z_{n}\right)\right|\right) d x \leq \varepsilon_{6}(n) .
\end{aligned}
$$

Which gives by using 22,

$0 \leq \sum_{i=1}^{N} \int_{\Omega}\left(a_{i}\left(x, T_{k}\left(u_{n}\right), \nabla T_{k}\left(u_{n}\right)\right)-a_{i}\left(x, T_{k}\left(u_{n}\right), \nabla T_{k}(u)\right)\right)$

$\left(\frac{\partial T_{k}\left(u_{n}\right)}{\partial x_{i}}-\frac{\partial T_{k}(u)}{\partial x_{i}}\right) \leq 2 \varepsilon_{6}(n)$

then lemma 2 gives

$$
T_{k}\left(u_{n}\right) \rightarrow T_{k}(u) \text { strongly in } W_{0}^{1, \vec{p}}(\Omega) .
$$

\subsection{Existence results}

Theorem 1 Assume that $\bar{p}<N$ and (5)- 12 hold. Then there exists at least a weak solution of the problem (1).

Proof: By $(4.2)$ the sequence $\left(\frac{\partial u_{n}}{\partial x_{i}}\right)_{n}$ is bounded in $L^{p_{i}}(\Omega)$, so we have that $\frac{\partial u_{n}}{\partial x_{i}} \rightarrow \frac{\partial u}{\partial x_{i}}$ weakly in $L^{p_{i}}(\Omega)$ for $i=1, \ldots, N$ and $u_{n} \rightarrow u$ strongly in $L^{p_{-}}(\Omega)$. By 27) there exists a subsequence, which we still denote by $u_{n}$ such that $\frac{\partial u_{n}}{\partial x_{i}} \rightarrow \frac{\partial u}{\partial x_{i}} \quad$ a. e. in $\Omega$ for $i=1, \ldots, N$, then for $i=1, \ldots, N$, we have

$$
\left\{\begin{array}{l}
a_{i}\left(x, u_{n}, \nabla u_{n}\right) \rightarrow a_{i}(x, u, \nabla u) \text { a. e. in } \Omega, \\
g_{i}^{n}\left(x, u_{n}, \nabla u_{n}\right) \rightarrow g_{i}(x, u, \nabla u) \text { a. e. in } \Omega, \\
H_{i}^{n}\left(x, \nabla u_{n}\right) \rightarrow H_{i}(x, \nabla u) \text { a. e. in } \Omega .
\end{array}\right.
$$

Moreover by 6 and 10 , we have

$\int_{\Omega}\left|a_{i}\left(x, u_{n}, \nabla u_{n}\right)\right|^{p_{i}^{\prime}} \leq C\left[\int_{\Omega}\left|u_{n}\right|^{p_{\infty}}+\int_{\Omega}\left|\frac{\partial u_{n}}{\partial x_{i}}\right|^{p_{i}}\right] \quad$ and $\int_{\Omega}\left|H_{i}^{n}\left(x, \nabla u_{n}\right)\right|^{p_{i}^{\prime}} \leq C \int_{\Omega}\left|\frac{\partial u_{n}}{\partial x_{i}}\right|^{p_{i}}$

by $14,\left(a_{i}\left(x, u_{n}, \nabla u_{n}\right)\right)_{n}$ and $\left(H_{i}\left(x, \nabla u_{n}\right)\right)_{n}$ are bounded in $L^{p_{i}}(\Omega)$ then $a_{i}\left(x, u_{n}, \nabla u_{n}\right) \rightarrow a_{i}(x, u, \nabla u)$ weakly in $L^{p_{i}^{\prime}}(\Omega)$ and $H_{i}\left(x, \nabla u_{n}\right) \rightarrow H_{i}(x, \nabla u)$ weakly in $L^{p_{i}^{\prime}}(\Omega)$. Now we prove that $g_{i}^{n}\left(x, u_{n}, \nabla u_{n}\right)$ is uniformly equiintegrable for $i=1, \ldots, N$. For any measurable $E$ of $\Omega$ and for any $k \in \mathbb{R}^{+}$, we have

$\int_{E}\left|g_{i}^{n}\left(x, u_{n}, \nabla u_{n}\right)\right|$
$=\int_{E \cap\left\{\left|u_{n}\right| \leq k\right\}}\left|g_{i}^{n}\left(x, u_{n}, \nabla u_{n}\right)\right|+\int_{E \cap\left\{\left|u_{n}\right|>k\right\}}\left|g_{i}^{n}\left(x, u_{n}, \nabla u_{n}\right)\right|$
$\leq \int_{E \cap\left\{\left|u_{n}\right| \leq k\right\}} L(k)\left|\frac{\partial T_{k}\left(u_{n}\right)}{\partial x_{i}}\right|^{p_{i}}+\int_{E \cap\left\{\left|u_{n}\right|>k\right\}}\left|g_{i}^{n}\left(x, u_{n}, \nabla u_{n}\right)\right|$,
for fixed $k$ and for $i=1, \ldots, N$. For the first term we recall $\frac{\partial T_{k}\left(u_{n}\right)}{\partial x_{i}}$ strongly converges to $\frac{\partial T_{k}(u)}{\partial x_{i}}$ in $L^{p_{i}}(\Omega)$ for $i=1, \ldots, N$. Taking $T_{k}\left(u_{n}\right)$ as test function in (4.1), then $\sum_{i=1}^{N} \int_{\Omega} a_{i}\left(x, u_{n}, \nabla u_{n}\right) \frac{\partial T_{k}\left(u_{n}\right)}{\partial x_{i}}+\sum_{i=1}^{N} \int_{\Omega} g_{i}^{n}\left(x, u_{n}, \nabla u_{n}\right) T_{k}\left(u_{n}\right)+$ $\sum_{i=1}^{N} \int_{\Omega} H_{i}^{n}\left(x, \nabla u_{n}\right) T_{k}\left(u_{n}\right)=\int_{\Omega} f T_{k}\left(u_{n}\right)+\sum_{i=1}^{N} \int_{\Omega} k_{i} \frac{\partial T_{k}\left(u_{n}\right)}{\partial x_{i}}$, which implies that

$\sum_{i=1}^{N} \int_{\Omega} g_{i}^{n}\left(x, u_{n}, \nabla u_{n}\right) T_{k}\left(u_{n}\right) \leq\left(\int_{\Omega}|f|^{p_{\infty}^{\prime}}\right)^{\frac{1}{p_{\infty}^{\prime}}}\left(\int_{\Omega}\left|u_{n}\right|^{p_{\infty}}\right)^{\frac{1}{p_{\infty}}}+$ $\sum_{i=1}^{N}\left(\int_{\Omega}\left|k_{i}\right|^{p_{i}^{\prime}}\right)^{\frac{1}{p_{i}^{\prime}}}\left(\int_{\Omega}\left|\frac{\partial u_{n}}{\partial x_{i}}\right|^{p_{i}}\right)^{\frac{1}{p_{i}}}+$

$$
\begin{gathered}
\sum_{i=1}^{N} \int_{\Omega} b_{i}\left|\frac{\partial u_{n}}{\partial x_{i}}\right|^{p_{i}-1}\left|T_{k}\left(u_{n}\right)\right| \\
\leq\left(\int_{\Omega}|f|^{p_{\infty}^{\prime}}\right)^{\frac{1}{p_{\infty}^{\prime}}}\left(\int_{\Omega}\left|u_{n}\right|^{p_{\infty}}\right)^{\frac{1}{p_{\infty}}}+ \\
\sum_{i=1}^{N}\left(\int_{\Omega}\left|k_{i}\right|^{p_{i}^{\prime}}\right)^{\frac{1}{p_{i}^{\prime}}}\left(\int_{\Omega}\left|\frac{\partial u_{n}}{\partial x_{i}}\right|^{p_{i}}\right)^{\frac{1}{p_{i}}}+ \\
\sum_{i=1}^{N}\left(\int_{\Omega} b_{i}^{r_{i}}\right)^{\frac{1}{r_{i}}}\left(\int_{\Omega}\left|\frac{\partial u_{n}}{\partial x_{i}}\right|^{p_{i}}\right)^{\frac{1}{p_{i}^{\prime}}}\left(\int_{\Omega}\left|u_{n}\right|^{p_{\infty}}\right)^{\frac{1}{p_{\infty}}}
\end{gathered}
$$

$\leq C_{1}$,

where $r_{i}$ is such that $\frac{1}{r_{i}}=\frac{1}{p_{i}}-\frac{1}{p_{\infty}}$. Let $E$ a measurable subset of $\Omega$ and for any $m \in \mathbb{R}^{+}$, we have

$\int_{E}\left|g_{i}^{n}\left(x, u_{n}, \nabla u_{n}\right)\right| d x$

$=\int_{E \cap\left\{\left|u_{n}\right| \leq k\right\}}\left|g_{i}^{n}\left(x, u_{n}, \nabla u_{n}\right)\right| d x+$

$\int_{E \cap\left\{\left|u_{n}\right|>k\right\}}\left|g_{i}^{n}\left(x, u_{n}, \nabla u_{n}\right)\right| d x$
$\leq \int_{E \cap\left\{\left|u_{n}\right| \leq k\right\}} L(k)\left|\frac{\partial u_{n}}{\partial x_{i}}\right|^{p_{i}} d x+$

$\begin{aligned} & \int_{E \cap\left\{\left|u_{n}\right|>k\right\}}\left|g_{i}^{n}\left(x, u_{n}, \nabla u_{n}\right)\right| d x \\ \leq & \int_{E \cap\left\{\left|u_{n}\right| \leq k\right\}} L(k)\left|\frac{\partial T_{k}\left(u_{n}\right)}{\partial x_{i}}\right|^{p_{i}} d x+\end{aligned}$

$\frac{1}{k} \int_{\left\{\left|u_{n}\right|>k\right\}} T_{k}\left(u_{n}\right) g_{i}^{n}\left(x, u_{n}, \nabla u_{n}\right) d x$

we have $\frac{\partial T_{k}\left(u_{n}\right)}{\partial x_{i}} \rightarrow \frac{\partial T_{k}(u)}{\partial x_{i}}$ strongly in $L^{p_{i}}(\Omega)$ and $\int_{\left\{\left|u_{n}\right|>k\right\}} T_{k}\left(u_{n}\right) g_{i}^{n}\left(x, u_{n}, \nabla u_{n}\right) d x \leq C_{1}$

then $g_{i}^{n}$ is uniformly equi-integrable for any $i$, since $g_{i}^{n}\left(x, u_{n}, \nabla u_{n}\right) \rightarrow g_{i}(x, u, \nabla u)$ a. e. in $\Omega$, we get $g_{i}^{n}\left(x, u_{n}, \nabla u_{n}\right) \rightarrow g_{i}(x, u, \nabla u)$ in $L^{1}(\Omega)$. That allow us to pass to the limit in the approximate problem.

Remark 1 The condition (6) can be substituted by $\left|a_{i}(x, s, \xi)\right| \leq \gamma\left[j_{i}+|s|^{\frac{p_{\infty}}{p_{i}^{\prime}}}+\left|\xi_{i}\right|^{p_{i}-1}\right]$, where $j_{i}$ is a positive function in $L^{p_{i}^{\prime}}(\Omega)$ for $i=1, \ldots, N$, and the condition (9) can be substituted by $\left|g_{i}(x, s, \xi)\right| \leq L(|s|)\left(C_{i}+\left|\xi_{i}\right|^{p_{i}}\right)$ where $C_{i}$ is a positive function in $L^{1}(\Omega)$ for $i=1, \ldots, N$. 


\section{References}

1. A. Alvino, M.F. Betta, A. Mercaldo, "Comparison principle for some classes of nonlinear elliptic equations.", J. Differential Equations., 12, 3279-3290, 2010. http://dx.doi.org/10.1016/j.jde.2010.07.030

2. S. Antontsev, M. Chipot, "Anisotropic equations: uniqueness and existence results.", Differential Integral Equations 21 ( 5), 401-419, 2008. https://projecteuclid.org/euclid.die/1356038624

3. L. Boccardo, T. Gallouét, P. Marcellini,“ Anisotropic equations in $L^{1}$.", Differential Integral Equations., 9(1), 209-212, 1996.

4. G, Bottaro, M. Marina,“ Problema di Dirichlet per equazioni ellittiche di tipo variazionale su insiemi non limitati.", Boll. Un. Mat. Ital, 8, 46-56, 1973.

5. F. Li, "Anisotropic elliptic equations in $\mathrm{L}^{m}$.", J. Convex Anal, 8 (2), 417-422, 2001.

6. O. Guibé, A. Mercaldo," Uniqueness results for non coercive nonlinear elliptic equations with two lower order terms.", Commun. Pure Appl. Anal. 7 (1), 163-192, 2008.

7. V. Monetti, L. Randazzo, “ Existence results for nonlinear elliptic equations with p-growyh in the gradient.", Ricerche di Matematica 1,. 163-181, 2000.
8. Y. Akdim, A. Benkirane, M. El Moumni, “ Existence results for nonlinear elliptic problems with lower order terms.", International Journal of Evolution Equations (IJEE)., 4, 1-20, 2014.

9. R. Di Nardo, F. Feo, "Existence and uniqueness For nonlinear anisotropic elliptic equations.", Arch. Math.102 141-153, 2014.

10. Y. Akdim, A. Salmani, “ Existence And Uniqueness Results for nonlinear Anisotropic Elliptic Equations.", Journal of Nonlinear Evolution Equations and Applications (JNEEA)., 6, 95-111, 2016.

11. A. Di Castro,"Existence and regularity results for anisotropic elliptic problems.", Adv. Nonlinear Stud, 9, 367-393, 2009.

12. M.Troisi,“ Teoremi di inclusione per spazi di Sobolev non isotropi.", recerche Mat.18, 3-24, 1969.

13. I. Fragala, F. Gazzola, B. Kawohl,“ Existence and nonexistence results for anisotropic quasi- linear elliptic equations.", Ann. Inst. H. Poincar Anal. Non Linéaire, 21(5), 715-734, 2004.

14. J. Leray, J. L. Lions," Quelques rsultats de Visik sur les problémes elliptiques nonlinéaires par les méthodes de Minty-Browder.", Bull. Soc. Math. France 93 97-107, 1965.

15. L. Boccardo, F. Murat, J. P. Puel,“ Existence of bounded solution for non linear elliptic unilateral problems.", Ann. Mat. pura appl., 152, 183-196, 1988. 